\title{
3 Simultaneous Estimation by Partial Totals for Compartmental Models $C$
}
Ł John J. Beauchamp and Richard G. Corne $11 \%$ | February 1, $1967 \mid 0$

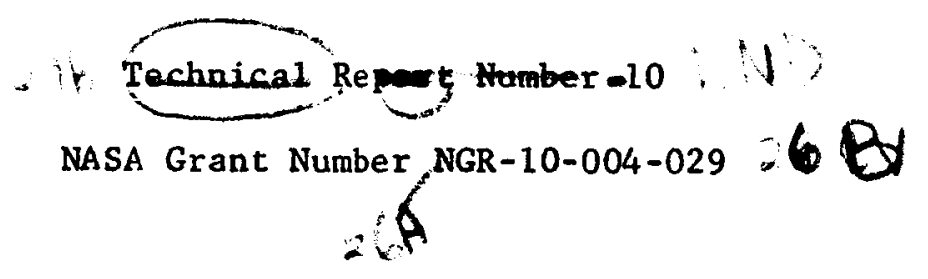

\footnotetext{
- Department of Statistics 3

Florida State University GTallahassee, Florida -
} 


\section{SIMULTANEOUS ESTIMTION BY PARTIAL TOTALS}

\section{FOR COMPARTMENTAL MODELS*}

John J. Beauchamp

Oak Ridge National Laboratory

and

Richard G. Cornell

Florida State University

An estimation procedure is presented that may be used to simultaneously estimate the parameters in a multiple equation regression model. The regression models considered are shown to arise from radioactive tracer experiments using compartmental models. For such models the regression equations are shown to be linear combinations of the same exponential parameters, and the number of independent regression equations is also shown to be the same as the number of exponential parameters.

The estimation procedure is developed under the assumption of equally spaced values of the independent variable. The first step of the procedure involves the simultaneous estimation of the exponential parameters by a generalized patial totals approach, and the second step involves the simultaneous estimation of the linear parameters using the estimates of the nonlinear parameters. Modifications are presented that make the estimation procedure more versatile, and asymptotic properties are also investigated. The procedure is 1llustrated with generated data.

*This Investigation was part of the doctoral research of John $\mathrm{J}$. Beauchamp at Florida State University. It was supported by Public Health Service fellowship 5-F1-GM-15,632-03 and training grant 5T1 GM-913 from the National Institute of General Medical Sciences, and by NASA 


\section{INTRODUCTION}

In this paper we present and evaluate some of the properties of an estimation procedure which can be used to estimate the parameters present in the following set of $n$ regression equations:

$$
Y_{i j}=\alpha_{i 0}+\sum_{k=1}^{n} \alpha_{i k} e^{-\lambda x_{j}}+\varepsilon_{i j}
$$

for $1=1,2, \ldots, n$ and $j=0,1, \ldots, N-1$. In this expression $Y_{i j}$ and $E_{i j}$ represent random variables associated with the $j^{\text {th }}$ observation on the $i^{\text {th }}$ equation; $x_{j}$ is an independent variable; and the $\alpha_{1 k}$ 's and $\lambda_{k}$ 's are constant parameters that we estimate. The estimation procedure developed in Section 3 is for equally spaced values of $x_{j}$. In Section 2 we show that certain compartmental models for radioactive tracer experiments give rise to regression equations like (1.1).

Since the exponential parameters $\lambda_{1}, \lambda_{2}, \ldots, \lambda_{n}$ appear in each one of the regression equations of (1.1), we make use simultaneously of all of the observations on all of the equations being studied to estimate these parameters. Beauchamp and Cornell [2], Turner et al [9], and Zellner [10] present simultaneous least squares estimation procedures. However, Zellner only considers linear regression equations, Turner et al assume that the covariance matrix of the $\varepsilon_{1 j}$ is known, and the iterative procedures in [2] and [9] are often difficult to compute. The procedure presented here provides a simple alternative to the least squares procedures or can be used to compute initial estimates for such procedures. The estimation procedure presented in Section 3 is a generalization of the partial totals technique given by Cornell [5], who considered the estimation problem for a single regression equation that is a 
linear combination of exponential terms. In Section 4 some of the properties of the procedure presented in Section 3 are investigated and some modifications are given which will make the procedure more versatile. An example showing the application of the procedure is given in Section 5 .

\section{MODELS}

The use of radioactive tracers in biological investigations is an example of an experimental situation which yields data that may be reasonably described by the set of regression equations given in (1.1). Berman and Schoenfeld [3] and Sheppard [7] have discussed the formulation of mathematical models for such experiments. These formulations represent an organism by several chemical states or sites of a physiological substance designated as compartments. It is assumed that there are fixed transition probabilities or turnover rates from one compartment to another, and the whole system is assumed to be in steady state. The turnover rates are assumed to be proportional to the amounts of material in the compartments. The concept of dividing a biological system into a number of fixed compartments in merely an aid in analysis, since the various states or sites contain finer structure. However, the compartmental analysis does prove itself useful in understanding some of the mechanics of the system.

The mainmillary and catenary systems, which are discussed in detail in the work by Sheppard [7], are particular examples of such compartmentalized systems and are specified as follows:

1) The catenary system Involves $(n+1)$ compartments that may be thought of as arranged in a chain-1ike manner where each compartment has non-zero transition 
rates only with the compartments adjacent to it.

2) The mammillary system involves $n$ peripheral compartments that have turnover rates with a central compartment but no turnover between the $\mathrm{n}$ peripheral compartments.

From the discussion in the preceding paragraphs, the following set of differential equations is formed to describe the general $(n+1)$ - compartment problem:

$$
\frac{d E\left(Y_{i}(x)\right)}{d x}=-\tau_{i i} E\left(Y_{i}(x)\right)+\sum_{\substack{h=1 \\ h \neq 1}}^{n+1} \tau_{i h} E\left(Y_{h}(x)\right)
$$

for $1=1,2, \ldots, n+1$, where $E\left(Y_{i}(x)\right)$ is the expected amount of labeled material in the $i^{\text {th }}$ compartment at time $x, \tau_{i h}$ is the fractional amount of material in the $h^{\text {th }}$ compartment flowing to the $i^{\text {th }}$ compartment per unit time, and

$$
\tau_{i 1}=\sum_{\substack{h=1 \\ h \neq i}}^{n+1} \tau_{h i} .
$$

Berman and Schoenfeld [3] show that the solution of (2.1) is given by

$$
E\left(Y_{i}(x)\right)=\sum_{k=0}^{n} \alpha_{i k} e^{-\lambda k^{x}}
$$

for $i=1,2, \ldots, n+1$ where the constants $\alpha_{i k}$ are functions of the $\tau_{i h}$ and the initial conditions of the experiment. Let $\tau$ be an $(n+1) \times(n+1)$ matrix whose dlagonal elements are given by $\tau_{i i}$ and off diagonal elements are given by ${ }^{-\tau}{ }_{\text {ih }}$. Then $\lambda_{0}, \lambda_{1}, \ldots, \lambda_{n}$ are the characteristic roots of $\tau$. Throughout this paper we assume that the characteristic roots of $\tau$ are real and distinct.

From equation $(2.2)$ we note that the number of exponential terms in each equation is determined by the number of non-zero characteristic roots of 
the matrix $\tau$, which is either $n$ or $n+1$ when these roots are distinct and which is also equal to the rank of $\tau$. By writing down the matrix $\tau$ for the general $(n+1)$ - compartment catenary and mamillary systems, we can show that the rank of $\tau$ is equal to $n$, so exactly one $\lambda$, say $\lambda_{0}$, is zero. In addition, for the case when the amount of tracer material in the system is known and fixed, we can express the system of equations given in equation (2.2) in terms of a new quantity which represents the proportion of labeled material in the compartments at a time $x$. For this case there are only $n$ independent equations in (2.2) since

$\mathrm{n}+1$

$\sum_{i=1} E\left(Y_{i}(x)\right)$

is fixed for all $x$. We may now combine the above results into the following theorem:

Theorem 1: The regression model used to describe the general $(n+1)$ - compartment catenary and mammillary systems, when a fixed and known amount of tracer material is present in these systems, is given by the model in equation (1.1). Thus the model given by equation (1.1) includes models arising in two classes of compartmental models of interest in tracer experiments.

During the development of the estimation procedure given in the next section, the only assumption that we need to make about the random variables $\varepsilon_{i f}$ is that $E\left(\varepsilon_{i j}\right)=0$ for all $i$ and $j$. However, additional assumptions are needed in order to investigate some of the properties of the estimators found by this procedure and these will be given in Section 4 . 


\section{PARTIAL TOTALS ESTIMATION}

In this section we present the partial totals estimation technique for the model in equation (1.1) for two cases.

Case I: The regression model is given by

$$
Y_{i j}=\sum_{k=1}^{n} \alpha_{i k} e^{-\lambda_{k} x_{j}}+\varepsilon_{i j},
$$

for $i=1,2, \ldots, n$ and $j=0,1, \ldots,(n+1) M-1$, where the observable random variable $Y_{i j}$ takes on values denoted by $y_{i j}$. Comparing (1.1) and (3.1) we note that $\alpha_{\text {io }}=0$ and $N=(n+1) M$.

Case II: The regression model is given by

$$
Y_{1 j}=\alpha_{i 0}+\sum_{k=1}^{n} \alpha_{1 k} e^{-\lambda_{k} x_{j}}+\varepsilon_{i j},
$$

for $1=1,2, \ldots, n$ and $j=0,1, \ldots,(n+2) M-1$, where the observable random variable $Y_{i f}$ takes on values denoted by $y_{1 j}$. Comparing (1.1) and (3.2) we note that $N=(n+2) M$.

In the above two cases we are assuming that $n$ and $M$ are positive integers, the coefficients $\alpha_{i k}$ are real numbers, and the exponents $\lambda_{k}$ are distinct positive real numbers. We want to take our observations at equally spaced values of the independent vartable $x_{j}$, so we also assume that $x_{j}=c_{j}$ where $c$ is a positive constant.

The estimation of the exponential parameters will involve the application of a partial totals approach similar to that presented by Cornell $[4,5]$. First we consider the estimation of the exponential parameters for the regression model given by Case $I$. We group the observations from each equation into $(n+1)$ groups each containing $M$ observations. Then the following 
partial totals are formed:

$$
\begin{aligned}
& \sum_{j=(q-1) M}^{q M-1} Y_{i j}=\sum_{j=(q-1) M}^{q M-1} \sum_{k=1}^{n} \alpha_{i k} e^{-\lambda_{k} X_{j}}+\sum_{j=(q-1) M}^{q M-1} \varepsilon_{i j} \\
& =\Sigma_{i q}+\underset{j=(q-1) M}{\sum_{i j}} \varepsilon_{i j}
\end{aligned}
$$

for $1=1,2, \ldots, n$ and $q=1,2, \ldots, n+1$ where

$$
\Sigma_{i q}=\sum_{j=(q-1) M}^{q M-1} E\left(Y_{i j}\right) .
$$

Now for each value of 1 and equally spaced values of $x_{j}$, Cornell [5] shows for each single regression equation that the following equation is satisfied:

$$
\Lambda_{n} \Sigma_{i 1}-\Lambda_{n-1} \Sigma_{i 2}+\Lambda_{n-2} \Sigma_{i 3}-\ldots+(-1)^{n} \Lambda_{0} \Sigma_{i, n+1}=0
$$

for $1=1,2, \ldots, n$ where the $\Lambda_{r}$ are the elementary symmetric functions of the $\exp \left(-\lambda_{k} c M\right), 1 . e ., \Lambda_{r}$ equals the sum of all possible products of the terms $\exp \left(-\lambda_{k} c M\right)$ taken $r$ at a time, $r=1,2, \ldots, n$. In addition, we define $\Lambda_{0}=1$. Therefore, since $i=1,2, \ldots, n$, in equation (3.4) we have $n$ equations In the $n$ unknowns $\Lambda_{r}$. Hence by substituting

$$
s_{i q}=\underset{f=(q-1) M}{q M-1} y_{i j}
$$

for $\Sigma_{\text {iq }}$ we may easily solve for estimators of $\Lambda_{r}$ which are denoted by $L_{r}$ * From these estimators of the elementary symmetric functions we may now obtain estimators of $\exp \left(-\lambda_{k} c M\right)$ for $k=1,2, \ldots, n$. Using the same properties of elementary symetric functions that Cornell [5] used for the single equation problem, the estimators of $\exp \left(-\lambda_{k} c M\right), k=1,2, \ldots, n$, are obtained by finding 
the $n$ roots of the polynomial equation

$$
w^{n}-L_{1} w^{n-1}+L_{2} w^{n-2}-\ldots+(-1)^{n} L_{n}=0
$$

Since the ordering of the exponential parameters is arbitrary we take the ordering to be $\lambda_{1}<\lambda_{2}<\ldots<\lambda_{n}$. Then the roots of (3.5) are denoted by $w_{1}>w_{2}>\ldots>w_{n}$ and the estimators of $\lambda_{k}$ aze given by $\hat{\lambda}_{k}=(-1 / c M)$ In $w_{k}$, $k=1,2, \ldots, n$.

For the Case II model given by equation (3.2), we group the observations into $(n+2)$ groups each containing $M$ observations, and then form the following partial totals: $S_{i q}=S_{i q}-S_{i, q+1}$ for $i=1,2, \ldots, n$ and $q=1,2, \ldots, n+1$, where the partial totals

$$
s_{i q}=\sum_{j=(q-1) M}^{q-1} y_{i j} .
$$

For each $S_{i q}^{i}$ there is a corresponding $\Sigma_{i q}^{\prime}=\Sigma_{i q}-\Sigma_{i, q+1}$, and Cornell [5] shows that the following equation is satisfied for each $i$ by the $\Sigma_{i q}$ :

$$
\Lambda_{n} \Sigma_{i 1}-\Lambda_{n-1} \Sigma_{i 2}^{i}+\Lambda_{n-2} \Sigma_{i 3}-\ldots+(-1)^{n} \Lambda_{0} \Sigma_{i, n+1}=0,
$$

for $1=1,2, \ldots, n$. This set of equations is the same as $(3,4)$ except tha. $\Sigma_{\text {iq }}^{\prime}$ 's have been substituted for $\Sigma_{1 q}$ 's. The $\Lambda_{r}, r=0,1,2, \ldots, n$, are the same as those defined earlier. We now proceed as in Case I to obtain esi-imators of the exponential parameters using the $\mathrm{S}_{i q}^{-}$'s instead of the $\mathrm{S}_{i q}$ 's, $i=1,2, \ldots, n$ and $q=1,2, \ldots, n+1$.

At this point we want to estimate the coefficients in the set of equations (3.1) and (3.2). To obtain these estimators we substitute the estimators of the $\lambda_{k}$, found by the partial totals procedure described above, 
into our set of $n$ independent equations. After doing this we have a set of $n$ regression equations which are linear in the unknown coefficients $\alpha_{i k}$. Therefore, to estimate these coefficients we use the weighted least squares procedure as given by Zellner [10] if it is reasonable to assume that the covariance matrix of the random variables $\varepsilon_{i f}$ is known apart from a constant multiplier. Otherwise, we apply a weighted least squares procedure using an estimated covariance matrix as discussed by Beauchamp and Cornell [2] and Telser [8]. So our estimation procedure involves two main steps: (1) the estimation of the exponential or nonlinear parameters by a generalized partial totals approach; and (2) the estimation of the coefficients or linear parameters by a weighted least squares approach after substituting the estimates of the nonlinear parameters into the regression equations. Sometimes only estimates of the exponential parameters are required in the analysis of compartmental data, in which case only the first of these steps would be applied.

\section{EVALUATION AND MODIFICATIONS}

From the presentation of the estimation procedure in the preceding section, it can easily be seen that the $S_{i q}$ and $S_{i q}$ could be replaced by the means $\bar{s}_{i q}=s_{i q} / M$ and $\bar{s}_{i q}^{\prime}=s_{i q}^{\prime} / M$, respectively, and the estimators of the exponential parameters would remain the same. By thinking of our estimators of the exponential parameters as functions of these sample means, we can demonstrate the consistency of these estimators as $M \rightarrow \infty$ under the following assumptions :

(1) For each value of $i=1,2, \ldots, n$, the random variables $\varepsilon_{i j}$, for all values of $j$, are uncorrelated with $E\left(\varepsilon_{1 j}\right)=0$. 
(2) For each value of $i$ and $q$ the random variables $\varepsilon_{i j}$ associated with the corresponding observations $y_{i j}$ in $S_{i q}$ or $S_{i q}^{j}$ as given in Section 3 have common variance.

(3) For each value of 1 and $q$ the domain of the independent variable is of constant length $J$ for $s_{i q}$ or $s_{i q}^{\prime}$ where $i=1,2, \ldots, n$ and $q=1,2, \ldots, n+1$. (4) For the linear coefficients $\alpha_{i k}$, let $\alpha$ be the $n \times n$ matrix of these coefficients, where $\alpha_{i k}$ is the element in the $i^{\text {th }}$ row and $k^{\text {th }}$ column for $1, k=1,2, \ldots, n$, and assume that the determinant of $\alpha$ is unequal to zero. The demonstration of the consistency as $M \rightarrow \infty$ of the exponential parameters of our regression model involves the application of the Tchebycheff theorem given in Cramer [6], and follows the same line of argument as used by Cornell $[4,5]$. Then the estimators of the linear parameters are also consistent if these estimators are continuous functions of the $\hat{\lambda}_{k}$ 's and if the covariance matrix of the $\varepsilon_{i j}$ 's is specified apart from a constant multiplier or is replaced by a consistent estimator in the weighted least squares calculations.

Under the four assumptions given at the beginning of this section plus the additional assumpticn that the estimators of the exponential parameters possess continuous second order devivatives of every kind with respect to the $\overline{\mathrm{s}}_{i q}$ or $\overline{\mathrm{s}}_{\hat{i q}}$, Beauchamp [1] demonstrates that $\sqrt{\mathrm{M}}(\hat{\lambda}-\lambda)$ has a limiting multivariate normal distribution, where $\hat{\lambda}$ represents the vector of estimators of the exponential parameters whose true values are given in the vector $\lambda$, as $M+\infty$. The mean vector of this limfing distribution is shown to be equal to the zero vector and, for Case $I$, the covariance matrix of this limiting distribution is shown to be equal to $\mathrm{F} \Omega \mathrm{F}^{\mathrm{T}}$ where 


$$
\begin{aligned}
\Omega & =M E\left(\bar{\varepsilon}_{* .} \bar{\varepsilon}_{* .}^{T}\right), \bar{\varepsilon}_{* \cdot}^{-11-}=\frac{1}{M} \sum_{j=0}^{M-1} \varepsilon_{* j}, \\
\varepsilon_{* j} & =\left(\varepsilon_{1 j}, \varepsilon_{1, j+M}, \ldots, \varepsilon_{1, j+n M}, \varepsilon_{2 j}, \ldots, \varepsilon_{2, j+n M}, \ldots, \varepsilon_{n j}, \ldots, \varepsilon_{n, j+n M}\right)^{T},
\end{aligned}
$$

the superscript $T$ represents the transpose of a matrix or vector, and $R$ represents the $n \times n(n+1)$ matrix

$$
\left(\begin{array}{ccc}
\frac{\partial \hat{\lambda}_{1}}{\partial \bar{s}_{11}} & \cdots & \frac{\partial \hat{\lambda}_{1}}{\partial \bar{s}_{n, n+1}} \\
\vdots & & \vdots \\
\frac{\partial \hat{\lambda}_{n}}{\partial \bar{s}_{11}} & \cdots & \frac{\partial \hat{\lambda}_{n}}{\partial \bar{s}_{n, n+1}}
\end{array}\right)
$$

evaluated at the point $S_{i q}=\psi_{i q}$ for all $i$ and $q$ where $S_{i q}$ converges in probability to

$$
\psi_{1 q}=\frac{1}{J} \sum_{k=1}^{n} \frac{\alpha_{i k}}{\lambda_{k}} e^{-\lambda_{k}(q-1) J}\left(1-e^{-\lambda_{k} J}\right) \text {. A similar result holds for }
$$

Case II.

In the above discussion the estimation procedure was developed assuming only one observation for different values of $x_{j}$. However, each $y_{i j}$ can be replaced by the mean of the observations for each value of $x_{j}$ without changing the estimation equations given earlier. If some of the values of $x_{j}$ in equations (3.1) and (3.2) are not equally spaced or if some of the number of values of $x_{j}$ taken for each partial total $s_{i q}$ are unequal, then we think of the $\Sigma_{i q}$ 's as approximations to areas under the curve found by plotting $E\left(Y_{1}(x)\right)$ against $x$ and suggest the following modifications to the 
estimation procedure:

(1) Divide the domain of the independent variable into the desired number of intervals, each interval being the same length.

(2) Add the observations in each of these new intervals together, weighting each observation $y_{i j}$ by $\left(x_{j+-1}-x_{j-1}\right) / 2$.

(3) Divide these new partial totals by the sum of the weights, and substitute these weighted averages in the place of the $\overline{\mathrm{s}}_{i q}$ 's given earlier. In order for the limiting properties of the estimators to hold, we must continue to assume that the domain of the independent variable for each partial total remains constant as the number of observations in each partial total becomes large. The above modifications are useful for the estimation of the exponential parameters. The estimation of the linear parameters, given the exponential estimates, remains unchanged.

\section{EXAMPLE}

In this section we apply the estimation procedure developed in Section 3 to the set of data given in Table 1 . The data given in this table were manufactured for the regression model given in equation (5.1) by adding random deviates to calculated expected values. The model given by (5.1) is a special case of (1.1) which arises by observing the proportions of radioactive tracer present in two out of the three compartments of either a catenary or mammillary system. The regression model of interest is given as follows:

$$
\begin{aligned}
& Y_{1 j}=\alpha_{1} e^{-\lambda_{1} x_{j}}+\left(1-\alpha_{1}\right) e^{-\lambda_{2} x_{j}}+\varepsilon_{1 j}, \\
& Y_{2 j}=1-\alpha_{2} e^{-\lambda_{1} x_{j}}-\left(1-\alpha_{2}\right) e^{-\lambda_{2} x_{j}}+\varepsilon_{2 j} .
\end{aligned}
$$


TABLE 1---Data to be fitted by generalized partial totals estimation procedure

\begin{tabular}{|c|c|c|c|}
\hline$x_{j}(=j)$ & $y_{1 j}$ & $y_{2 j}^{\prime}=1-y_{2 j}$ & Partial Totals \\
\hline 0 & 0.99580 & 0.98526 & \multirow{3}{*}{$s_{11}=5.23783$} \\
\hline 1 & 0.86755 & 0.90118 & \\
\hline 2 & 0.75378 & 0.78387 & \\
\hline 3 & 0.68462 & 0.72374 & \multirow[t]{5}{*}{$\mathrm{S}_{21}=5.63595$} \\
\hline 4 & 0.58998 & 0.64451 & \\
\hline 5 & 0.49806 & 0.58602 & \\
\hline 6 & 0.49066 & 0.57477 & \\
\hline 7 & 0.35738 & 0.43660 & \\
\hline 8 & 0.31896 & 0.44126 & \multirow{8}{*}{$S_{12}=1.96023$} \\
\hline 9 & 0.32844 & 0.43487 & \\
\hline 10 & 0.24684 & 0.34459 & \\
\hline 11 & 0.29593 & 0.38054 & \\
\hline 12 & 0.18045 & 0.28662 & \\
\hline 13 & 0.25398 & 0.33810 & \\
\hline 14 & 0.17297 & 0.29868 & \\
\hline 15 & 0.16266 & 0.28096 & \\
\hline 16 & 0.15076 & 0.24881 & \multirow{3}{*}{$s_{13}=0.95647$} \\
\hline 17 & 0.12821 & 0.22204 & \\
\hline 18 & 0.12233 & 0.24219 & \\
\hline 19 & 0.15341 & 0.29722 & \multirow[t]{5}{*}{$S_{23}=1.82865$} \\
\hline 20 & 0.13334 & 0.24112 & \\
\hline 21 & 0.08309 & 0.17590 & \\
\hline 22 & 0.09083 & 0.19781 & \\
\hline 23 & 0.09450 & 0.20356 & \\
\hline
\end{tabular}




\section{$-14-$}

Instead of recording the observations $y_{2 j}$ on the second regression equation of (5.1), we record $y_{2 j}^{\prime}=1-y_{2 j}$ so that

$$
E\left(Y_{2 j}\right)=\alpha_{2} e^{-\lambda_{1} x_{j}}+\left(1-\alpha_{2}\right) e^{-\lambda{ }^{x} j}
$$

is of the same functional form as $E\left(Y_{1 j}\right)$. In this example, $x_{j}=j$, $j=0,1, \ldots, 23$, and we have a Case I model with $n=2$ since we have two exponential parameters. Therefore, $M=8$ from (3.3), and we divide each set of observations into three groups each containing eight observations. Then we form the partial totals

$$
s_{i q}=\underset{j=8(q-1)}{8 q-1} y_{i j},
$$

for $i=1,2$ and $q=1,2,3$. The values of these partial totals are given in the last column of Table 1. Using equation (3.4) we are now able to use the set of equations given below to obtain estimates of the elementary symmetric functions of $\exp \left(-8 \lambda_{1}\right)$ and $\exp \left(-8 \lambda_{2}\right)$, denoted by $L_{1}$ and $L_{2}$ :

$$
\begin{aligned}
& S_{11} L_{2}-S_{12} L_{1}=-S_{13} \\
& S_{21} L_{2}-s_{22} L_{1}=-S_{23}
\end{aligned}
$$

or

$$
\begin{aligned}
& 5.23783 \mathrm{~L}_{2}-1.96023 \mathrm{~L}_{1}=-0.95647 \\
& 5.63595 \mathrm{~L}_{2}-2.80562 \mathrm{~L}_{1}=-1.82865 .
\end{aligned}
$$

Solving the set of equations given in (5.4), we find $L_{1}=1.14810$ and $L_{2}=0.24706$. Estimates of $\exp \left(-8 \lambda_{1}\right)$ and $\exp \left(-8 \lambda_{2}\right)$ are found by obtaining 
the roots of the quadratic equation

$$
w^{2}-1.14810 w+0.24706=0
$$

The roots of $(5.5)$ are given by $v_{1}=0.86123$ and $v_{2}=0.28687$. Therefore, as our estimates of $\lambda_{1}$ and $\lambda_{2}$ we take $\hat{\lambda}_{1}=[-\ln (0.86123)] / 8=0.01868$ and $\hat{\lambda}_{2}=[-\ln (0.28687)] / 8=0.15609$, respectively.

The next step in our estimation procedure is to estimate the linear parameters, $\alpha_{1}$ and $\alpha_{2}$. The observations in Table 1 were generated by adding random normal variables to calculated expected values and taking $E\left(\varepsilon_{1 j}^{2}\right)=\sigma_{11}=0.001 ; E\left(\varepsilon_{2 j}^{2}\right)=\sigma_{22}=0.001$; and $E\left(\varepsilon_{1 j} \varepsilon_{2 j}\right)=\sigma_{12}=0.0009$ for all $\mathrm{j}$. Therefore in this particular example we may assume that the covariance matrix $\Omega$ of the random variables $\varepsilon_{i j}$ is known. We may rewrite our regression model in equation (5.1) as

$$
\begin{aligned}
& Y_{1 j}-e^{-\lambda{ }^{x} j}=\alpha_{1}\left(e^{-\lambda_{1} x_{j}}-e^{-\lambda_{2} x_{j}}\right)+\varepsilon_{1 j}, \\
& 1-Y_{2 j}-e^{-\lambda} 2^{X_{1}}=\alpha_{2}\left(e^{-\lambda_{1} x_{j}}-e^{-\lambda} 2^{x_{j}}\right)+\varepsilon_{2 j} \text {. }
\end{aligned}
$$

If $\lambda_{1}$ and $\lambda_{2}$ were known, then the usual weighted least squares estimators of $\alpha_{1}$ and $\alpha_{2}$ would be given by

$$
\begin{aligned}
& \hat{\alpha}_{\star *}=\left(D_{Z}^{T} \Omega^{-1} D_{Z}\right)^{-1}\left(D_{Z}^{T} \Omega^{-1} y_{* *}\right)=\left(\hat{\alpha}_{1}, \hat{\alpha}_{2}\right)^{T}, \\
& D_{z}=\left(\begin{array}{ll}
Z & 0 \\
0 & z
\end{array}\right) \text {, } \\
& Z=\left(0, e^{-\lambda_{1}}-e^{-\lambda_{2}}, \ldots, e^{-23 \lambda_{1}}-e^{-23 \lambda_{2}}\right)^{T}, \Omega=\sigma_{* *} \otimes I, \sigma_{* *}=\left(\begin{array}{ll}
0.001 & 0.0009 \\
0.0009 & 0.001
\end{array}\right) \text {, }
\end{aligned}
$$

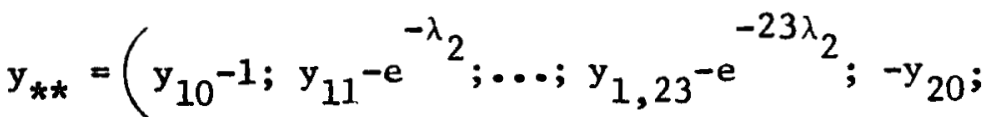

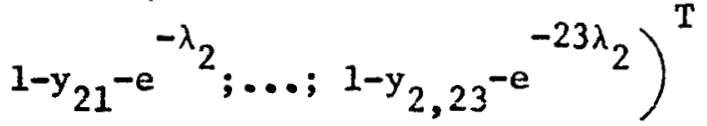


I is a $12 \times 12$ identity matrix, and $\otimes$ represents the Kronecker or direct product of two matrices. We now substitute the estimators $\hat{\lambda}_{1}$ and $\hat{\lambda}_{2}$ into equation (5.7) giving us the estimates of the parameters $\alpha_{1}$ and $\alpha_{2}$, denoted by $\hat{\alpha}_{1}=0.12880$ and $\hat{\hat{\alpha}}_{2}=0.25528$, respectively.

In case we do not know the true values of $\sigma_{11}, \sigma_{22}$, and $\sigma_{12}$, we substitute $\hat{\lambda}_{1}$ and $\hat{\lambda}_{2}$ into the regression equations and obtain the single equation least squares estimates of $\alpha_{1}$ and $\alpha_{2}$. These are given by $\hat{\alpha}_{1}=0.10900$ and $\hat{\alpha}_{2}=0.27569$. Using these estimates and the same procedure as given by Beauchamp and Cornel1 [2] or Telser [8], we find $\hat{\sigma}_{11}=0.00064$, $\hat{\sigma}_{22}=0.00074$ and $\hat{\sigma}_{12}=0.00058$. Substituting these estimates into the matrix $\Omega$ in (5.7), our new estimates of $\alpha_{1}$ and $\alpha_{2}$ are given by 0.14735 and 0.27007 , respectively. The results of these calculations are summarized in Table 2 . Graphs of the original data $(x)$ and the fitted regression equations of our model are given in Figures 1 through 4. 
$-17-$

TABLE 2--Estimates of parameters in equation (5.1)

Parameter $\quad$ Generalized Partial Totals

$\Omega$ Known $\quad \Omega$ Unknown

$\begin{array}{lll}\alpha_{1} & 0.12880 & 0.14735 \\ \alpha_{2} & 0.25528 & 0.27007 \\ \lambda_{1} & 0.01868 & 0.01868 \\ \lambda_{2} & 0.15609 & 0.15609\end{array}$




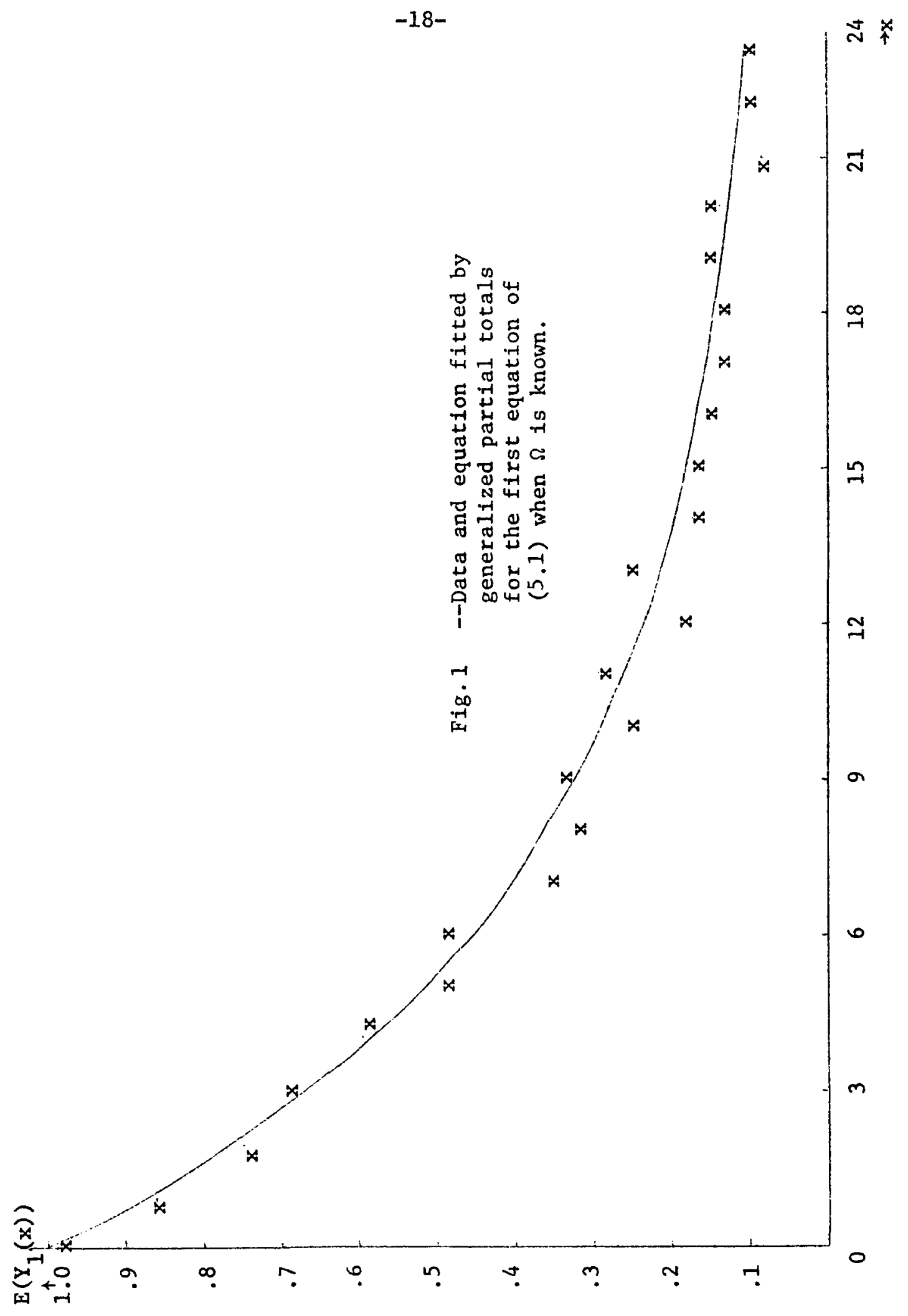




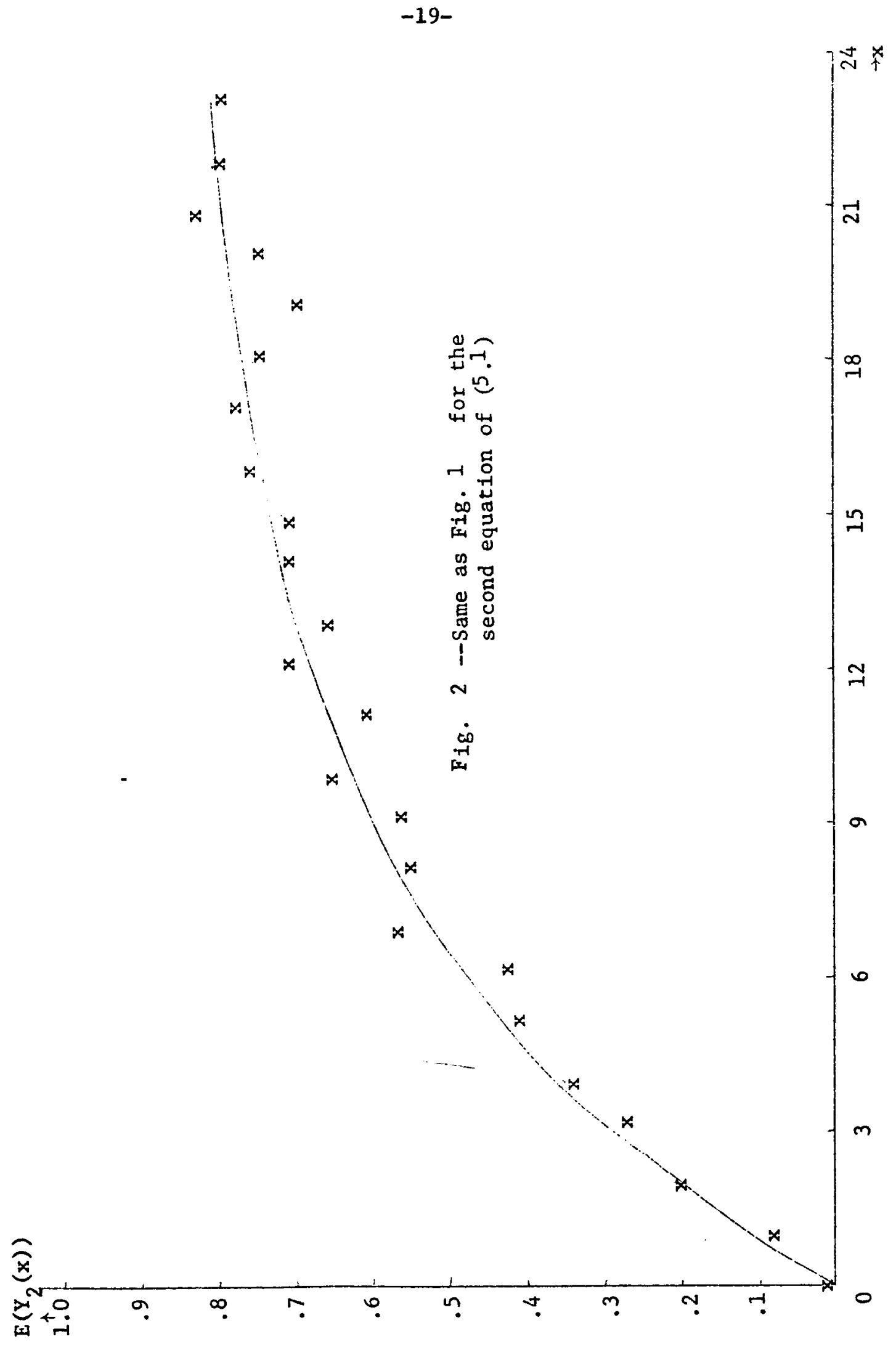




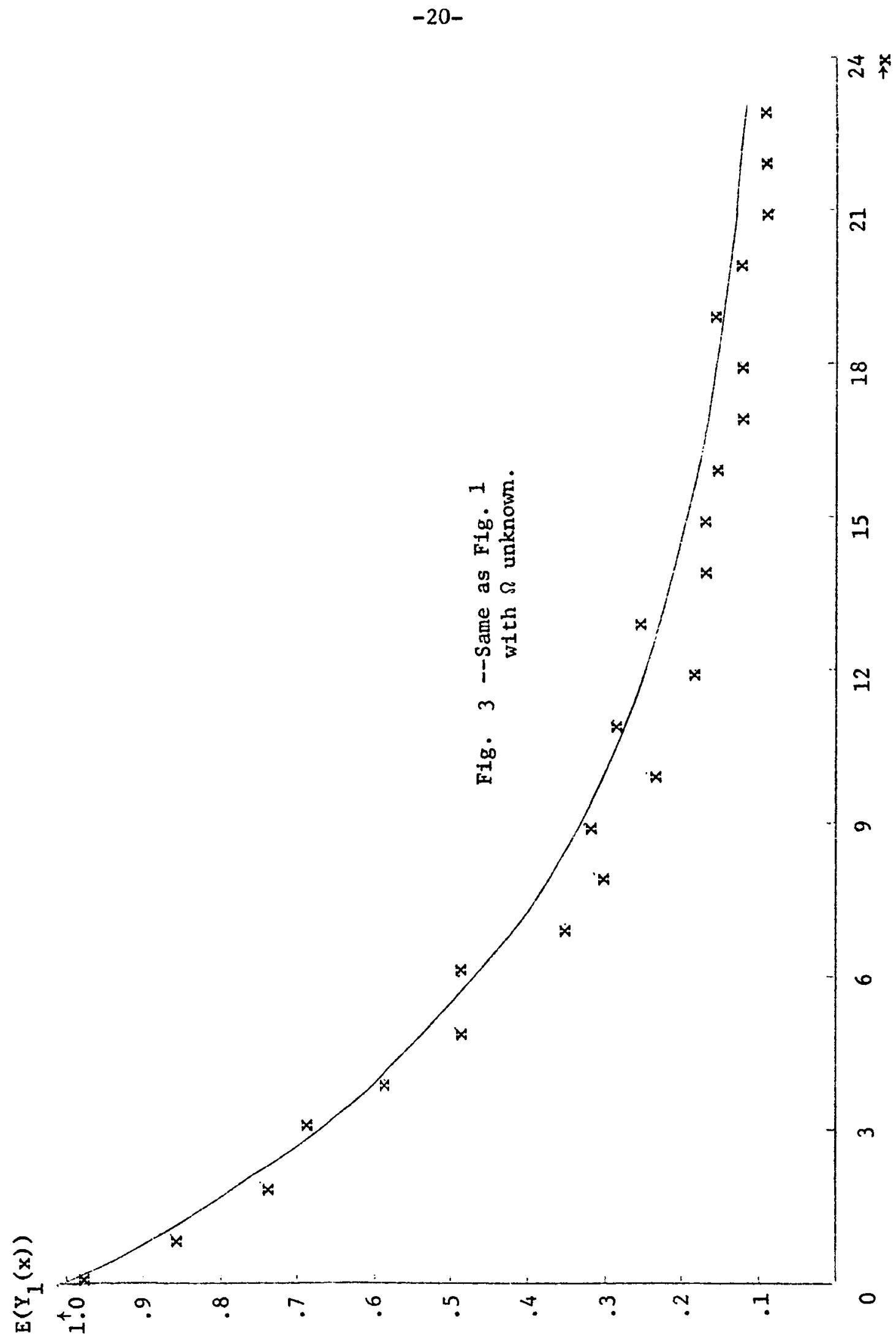




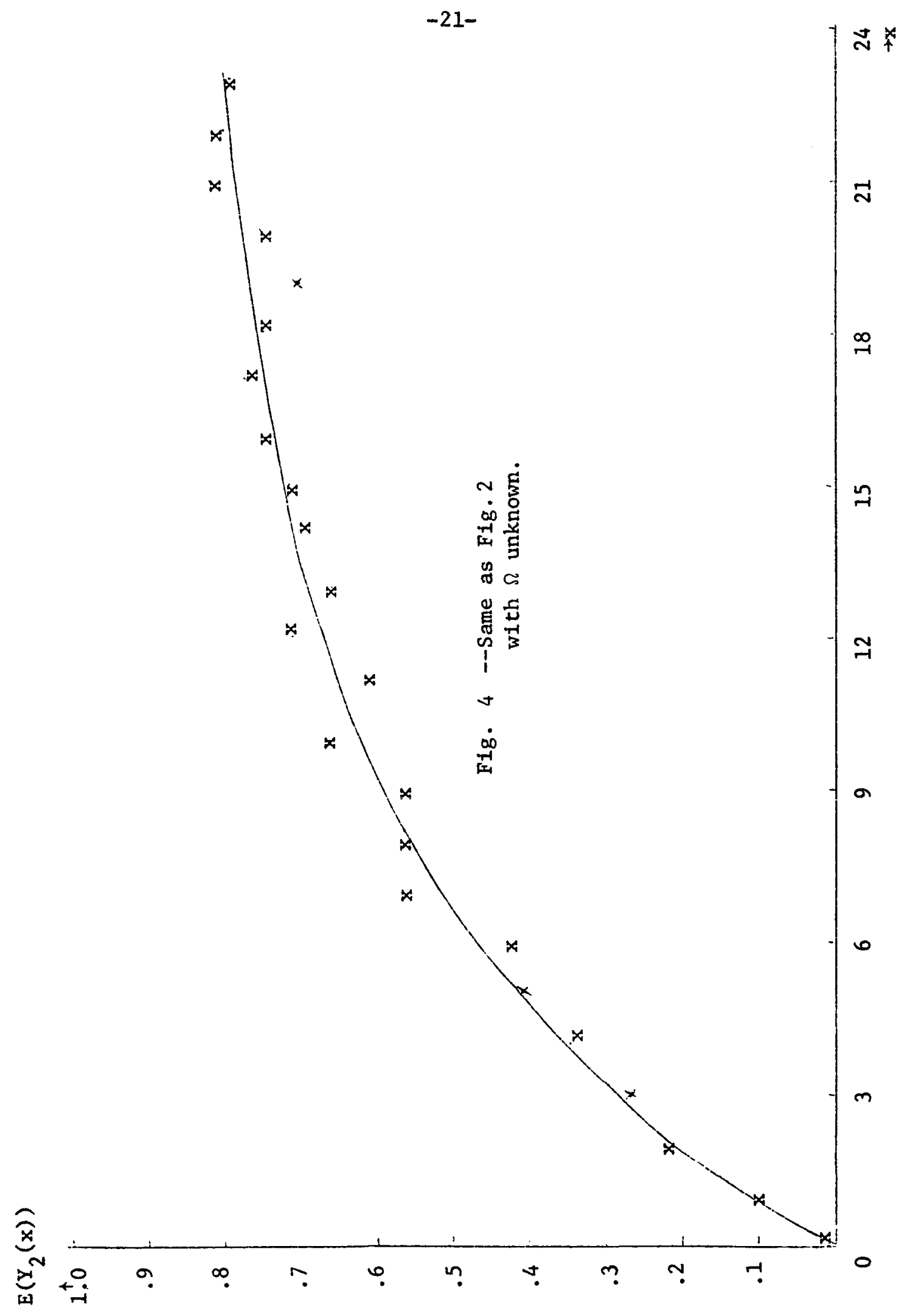


REFERENCES

[1] Beauchamp, J. J., Simultaneous Nonlinear Estimation, Ph.D. Dissertation, Florida State University, Tallahassee, Florda, 1966.

[2] Beauchamp, J. J., and Cornell, R. G., "Simultaneous nonlinear estimation," Technometrics, 8 (1966), 319-326.

[3] Berman, M., and Schoenfeld, R., "Invariants in experimental data on linear kinetics and the formation of models," Journal of Applied Physics, 27 (1956), 1361-1370.

[4] Cornel1, R. G., A New Estimation Procedure for Linear Combinations of Exponentials, Oak Ridge National Laboratory Report ORNL-2120, Oak Ridge, Tennessee, 1956.

[5] Cornell, R. G., "A method for fitting linear combinations of exponentials," Biometrics, 18 (1962), 104-113.

[6] Cramér, H., Mathematical Methods of Statistics, Princeton University Press, Princeton, New Jersey, 1946.

[7] Sheppard, C. W., Basic Principles of the Tracer Method, John Wiley and Sons, Incorporated, New York, New York, 1962.

[8] Telser, L. G., "Iterative estimation of a set of linear regression equations," Journal of the American Statistical Association, 59 (1964), 845-862.

[9] Turner, M. E., Monroe, R. J., and Homer, L. D., "Generalized kinetic regression analysis: hypergeometric kinetics," Biometrics, 19 (1963), 406-428.

[10] Zellner, A., "An efficient method of estimating seemingly unrelated regressions and tests for aggregation bias," Journal of the American Statistical Association, 57 (1962), 348-368. 